\title{
O ambiente escolar e as ações de promoção da saúde
}

\author{
The school environment and health promotion actions
}

Analie Nunes Couto,' William Vinicius Kleinpaul, ' Letícia Borfe, ${ }^{1}$ Sheila Cristina Vargas,

Hildegard Hedwig Pohl, ${ }^{1}$ Suzane Beatriz Frantz Krug ${ }^{1}$

"Universidade de Santa Cruz do Sul (Unisc), Santa Cruz do Sul, Rio Grande do Sul, Brasil.

Recebido em: 19/08/2016 / Aceito em: 27/09/2016 / Publicado em: 18/10/2016

analiecouto@hotmail.com

\section{RESUMO}

Objetivo: descrever as ações de promoção da saúde (PS) no ambiente escolar e as dificuldades que permeiam estas ações. Método: trata-se de uma revisão bibliográfica de caráter descritivo em publicações disponíveis na literatura científica sobre o tema, sem restrição de data. Utilizou-se pesquisa em sistemas de busca na internet, como o a Biblioteca Virtual em Saúde, com fontes indexadas nas bases de dados SciELO, LILACS, PubMed. Adotaram-se os descritores: Escolas Promotoras de Saúde, Saúde escolar, PS, em português e Health Promoting Schools, School health, Health promotion em inglês. Os artigos foram selecionados pelos títulos e resumos, após, lidos na íntegra e incluídos os com informações sobre o tema. Foram elaboradas categorias temáticas para a discussão dos dados encontrados, articulando-os com referenciais teóricos. Resultados: a PS busca fortalecer a ideia de autonomia dos sujeitos e dos grupos sociais, como um processo social e político, não limitado a abraçar ações direcionadas a fortalecer as habilidades e capacidades dos indivíduos. O ambiente escolar passou a ser reconhecido como apropriado para ações de PS, através de várias iniciativas de Escolas Promotoras da Saúde e do Programa Saúde na Escola, para ampliar os fatores protetores da saúde do escolar. No entanto, têm se destacado ações curativas e assistenciais e, as de PS têm sido confundidas com as de prevenção, influenciadas pelo antigo conceito. As ações apresentam como fragilidade a dificuldade na compreensão do conceito de PS pelos profissionais. Considerações finais: a efetividade da PS é um desafio metodológico e político que possibilita gerar conhecimento de programas adaptados à realidade de cada contexto, com vistas à aplicação de metodologia adequada. Para que as ações se concretizem de forma eficiente e permanente, é necessário o comprometimento de todos os envolvidos, propondo o empoderamento da comunidade escolar em atitudes do cotidiano.
Palavras-chave: Saúde na Escola; Promoção da Saúde; Escolas Promotoras de Saúde; Ambiente Escolar; Serviços de Saúde Escolar.

\section{ABSTRACT}

Objective: describe health promotion actions (HPA) in the school environment and the difficulties that permeate these actions. Method: this is a descriptive literature review in publications available in the scientific literature on the subject, with no date restriction. We surveyed in search engines on the Internet, such as the Virtual Health Library, with sources indexed in databases SciELO, LILACS, PubMed. We adopted the descriptors: Health Promoting Schools and School Health in Portuguese and Health Promoting Schools, School Health, Health promotion in English. The articles were selected by the titles and abstracts, read it fully and included with information on the subject. Themes for discussion of data were developed, linking them with theoretical frameworks. Results: HPA seeks to strengthen the idea of autonomy of individuals and social groups, as a social and political process, not limited to embrace actions directed at strengthening the skills and capabilities of individuals. The school environment has to be recognized as suitable for HPA actions through various initiatives Schools of Health Promotion and School Health Program, to extend the protective factors of school health. However, they have been outstanding curative and care actions and the HPA have been confused with the prevention, influenced by the old concept. Actions show how fragile the difficulty in understanding the concept of HPA by professionals. Closing remarks: the effectiveness of HPA is a methodological and political challenge that makes it possible to generate knowledge programs adapted to the reality of each context, with a view to the application of appropriate methodology. For the actions to be 
realized efficiently and permanently, the commitment of all stakeholders is necessary, proposing to empower the school community in everyday attitudes.

Keywords: Health in school; Health promotion; Health Promoting Schools; School Environment; School Health Services.

\section{INTRODUÇÃO}

Historicamente, as escolas de ensino representam espaços importantes utilizados para vivências e práticas em saúde. $\mathrm{O}$ espaço escolar tem sido utilizado para problematização e análise dos fatores determinantes das condições de saúde e doença, fundamentalmente quanto ao controle e prevenção do adoecimento, de situações de risco e agravos à saúde, através da vigilância epidemiológica e sanitária e assistência clínico-terapêutica, perdurando uma lógica higienista e preventivista. ${ }^{1}$

As ações de saúde escolar (ou higiene escolar) ganharam notoriedade a partir do ano de 1889, com ações sanitaristas, dadas as precárias condições de vida da sociedade, associadas à ausência de um sistema de saúde pública e a presença de epidemias. Estas foram desenvolvidas com a finalidade da diminuição da incidência de enfermidades. Somente a partir da década de 1950, os programas foram direcionados à saúde na escola com as ações voltadas às questões biológicas das problemáticas relacionadas à educação. Nesse período, havia expressa preocupação com a desnutrição, por ser relacionada como principal causa do fracasso escolar. Do mesmo modo, para justificar o baixo rendimento escolar, na década de 60 , os aspectos psicológicos, psiquiátricos, neurológicos e de saúde mental tiveram ênfase. ${ }^{2}$

A partir da década de oitenta, o ambiente escolar passou a ser reconhecido como apropriado para a promoção da saúde, através de várias iniciativas de Escolas Promotoras da Saúde. ${ }^{3}$ A Organização Mundial da Saúde define a promoção da saúde como um processo social e político, não limitado a abraçar ações direcionadas a fortalecerem as habilidades e capacidades dos indivíduos, mas envolvendo, também, ações dirigidas a mudar as condições sociais, ambientais e econômicas, de forma a amenizar o seu impacto na saúde pública e individual. A promoção parte de uma concepção positiva de saúde, ao estar mais preocupada com o bem-estar geral de pessoas e comunidades, possibilitando o desenvolvimento de uma reflexão crítica por parte dos diferentes atores sociais, uma vez que não adiantam políticas bem intencionadas se são inviabilizadas pela falta de vontade política e pela distribuição desigual de poder. ${ }^{4}$

A promoção da saúde traz, como um dos seus eixos, fortalecer a ideia de autonomia dos sujeitos e dos grupos sociais, e ao mesmo tempo, perspectivas progressistas, ressaltando a elaboração de políticas públicas intersetoriais, voltadas à melhoria da qualidade de vida das populações. ${ }^{5}$ Preconiza como necessário que os indivíduos ou coletividades sejam capazes de identificar e realizar aspirações, satisfazer necessidades, transformar e desenvolver mecanismos de adaptação ao meio ambiente para que tenha saúde, como um recurso para a vida cotidiana. Tem como objetivo desenvolver habilidades pessoais para viver a vida através do empowerment, e como estratégias a constituição de políticas públicas saudáveis, a criação de ambientes sustentáveis, a reorientação dos serviços de saúde, o fortalecimento de ações comunitárias e o desenvolvimento da capacidade individual. ${ }^{6}$

Na Conferência de Adelaide em 1988, a Carta de Compromisso com a Promoção da Saúde identifica cinco campos de ação para a promoção da saúde: construção de políticas públicas saudáveis, criação de ambientes favoráveis à saúde, desenvolvimento de habilidades, reforço da ação comunitária e reorientação dos serviços de saúde. Ainda, a temática da alimentação e nutrição foi citada como prioridade para a promoção de saúde, preconizando a criação de políticas públicas saudáveis pelos setores governamentais, garantindo ao consumidor acesso rápido a uma comida mais saudável (incluindo os estoques de alimentos em e para hospitais, escolas, abrigos e locais de trabalho). ${ }^{7}$

Através da mudança da concepção de saúde, houve uma modificação também do processo ensino-aprendizagem, levando em consideração suas interfaces, e a possibilidade da construção de uma nova perspectiva de educação e saúde. Foram desenvolvidos programas de saúde escolar, considerando as diferentes representações de concepções sobre saúde e sobre educação, o que contribuiu para refletir em práticas de saúde na escola. ${ }^{8}$

Para o alcance da promoção da saúde e o avanço da consolidação do SUS se fez necessária articulação das ações de saúde com as da educação, modificando a ideia de que a educação está associada apenas à escola, e a saúde somente aos serviços de saúde, superando as práticas isoladas, através de um trabalho integrado. O Ministério da Saúde (MS), por meio da Politica Nacional de Promoção da Saúde (PNPS), com o objetivo de integrar as ações de saúde ao currículo escolar, considerando os questionamentos da educação acerca da efetividade dessas ações e as mudanças conceituais e metodológicas no campo da saúde, recomenda a criação de espaços e ambientes saudáveis nas escolas. ${ }^{8,9}$

Articulado a isso, foram desenvolvidos programas e projetos indutores de políticas públicas, como os Programas Saúde na Escola e Saúde e Prevenção nas Escolas, a fim de ampliar a prevalência de fatores de proteção para a saúde do escolar. ${ }^{10} \mathrm{O}$ objetivo deste estudo é descrever as ações de promoção da saúde no ambiente escolar e as dificuldades que permeiam estas ações.

\section{MÉTODO}

Trata-se de uma revisão bibliográfica de caráter descritivo em publicações através do conhecimento disponíveis na literatura científica sobre o tema, sem restrição de data, por meio de pesquisa em sistemas de busca na internet, como o a Biblioteca Virtual em Saúde (BVS), com fontes primárias indexadas nas bases de dados SciELO, LILACS, PubMed. Adotou-se, para consulta às bases de dados, os seguintes descritores: Escolas Promotoras de Saúde, Saúde escolar, Promoção à saúde, em português e Health Promoting Schools, School health, Health promotion em inglês. Inicialmente, os artigos foram selecionados por meio de seus títulos e resumos, posteriormente os artigos foram lidos na íntegra e incluídos os que possuíam informações sobre 
o tema. Após, foram elaboradas categorias temáticas para a discussão dos dados encontrados, articulando-os com referenciais teóricos.

\section{Contextualização das ações de promoção da saúde no ambiente escolar}

Em 1995, com o compromisso de incentivar os países-membros da Organização Pan-Americana de Saúde (OPAS) houve o lançamento da Iniciativa Regional de Escolas Promotoras de Saúde, a fim de que fossem desenvolvidos programas e ações para favorecer a formação das futuras gerações de jovens. Estas tinham o objetivo de prover competências, destreza e conhecimento necessários à promoção e manutenção da saúde aos jovens, a partir de ações extensivas para a família e comunidade, com características transgeracional e construção de uma cultura de ambientes saudáveis na perspectiva da promoção da saúde. ${ }^{11}$

O tema promoção da saúde, como inclusão formal pela educação no Brasil, ocorreu no ano de 1996, a partir da aprovação da nova Lei de Diretrizes e Bases (LDB) da Educação Nacional, com a definição do planejamento e disposição da grade curricular e Parâmetros Curriculares Nacionais (PCNs) com questões referentes à saúde, ao meio ambiente, à orientação sexual, à ética, à pluralidade cultural, ao trabalho e consumo, e a outros temas importantes. ${ }^{2}$ Para que a escola seja denominada como Escola Promotora da Saúde deve propiciar um ambiente saudável, com relações harmônicas e construtivas, capazes de despertar aptidões e atitudes para a saúde nos participantes, promovendo a criatividade, a participação e a autonomia dos alunos e da comunidade escolar. ${ }^{12}$

As estratégias de saúde escolar devem criar espaços para o planejamento integrado da educação e da saúde, instigando a flexibilização dos currículos escolares com enfoque integral e participativo. A operacionalização de programas que promovam a melhoria da qualidade de vida pode ser incluída em discussões sobre a promoção e educação em saúde nas escolas, a fim de fortalecer a capacidade individual e social ao enfrentamento dos condicionantes da saúde e promover a participação, a autonomia e a criatividade de alunos, funcionários e professores nas discussões sobre a escola e o ambiente escolar. ${ }^{13}$

Para tanto, a concretização de ações de promoção da saúde no contexto escolar está apoiada nos professores. Com um papel de multiplicador de ideias, devem estar capacitados para abordar o conceito de saúde, através do domínio de informações e de estratégias educativas necessárias para a construção integrada do conhecimento preconizado nas Conferências Internacionais, Nacionais e nos Parâmetros Curriculares Nacionais (PCN). Torna-se necessário que o professor estimule a discussão de temas atuais, o reforço de habilidades que incentivem a criatividade, autonomia, a curiosidade o desejo de aprender nos alunos, através de metodologias pedagógicas como chave para o aprendizado. Neste processo, inclui-se a participação da comunidade escolar na seleção dos temas a serem trabalhados em sala de aula, através de discussão crítica das relações existentes entre as condições sociodemográficas do entorno, os comportamentos individuais e os riscos para a saúde. Quando os conteúdos escolares estão a serviço da vida e do bem viver ocorre o sentido do aprendizado e consequentemente a valorização dos processos afetivos e intelectuais. ${ }^{12,13}$

A OPAS, como estratégia de mobilizar a implantação desse modelo, procurou identificar experiências exitosas nas Américas e Europa, em realizações de concursos, premiou a experiência brasileira da escola municipal do Rio de Janeiro por incluir no projeto político pedagógico questões de saúde. ${ }^{14} \mathrm{O}$ Brasil apreciou as experiências regionais e locais, no Rio de Janeiro/ RJ, Embú/SP, Maceió/AL, Curitiba/PR e Palmas/TO, por valorizarem os contextos locais, interesses e desejos das comunidades e territórios, relevantes para a produção de conhecimento sobre saúde na escola. Entretanto, não chegou a instituir política de saúde na escola com base na EPS. ${ }^{1}$

Posteriormente, o Programa Nacional de Saúde na Escola (PSE), foi instituído por decreto presidencial em 2007, criando cenário favorável ao debate sobre a perspectiva de uma política nacional de saúde na escola como resultado do trabalho integrado entre Ministério da Saúde e Ministério da Educação. ${ }^{14}$ Este programa se propõe articular e integrar os dois setores com ações que buscam melhorar a qualidade de vida dos alunos de escolas de educação básica, tendo por base ações de prevenção, promoção e atenção à saúde, em articulação com a Estratégia Saúde da Família. ${ }^{2}$

Diferentemente do proposto pelas escolas promotoras de saúde que tinham ações de promoção da saúde apoiada nos professores, o PSE busca promover a comunicação entre escolas e unidades de saúde, assegurando a troca de informações sobre as condições de saúde dos estudantes. Procura, ainda, articular as ações da rede pública de saúde com as ações da rede pública de Educação Básica; promover a saúde e a cultura de paz, reforçando a prevenção de agravos à saúde; contribuir para a constituição de condições para a formação integral de educandos e para a construção de sistema de atenção social, com foco na promoção da cidadania e nos direitos humanos; fortalecer o enfrentamento das vulnerabilidades, no campo da saúde, que possam comprometer o pleno desenvolvimento escolar e fortalecer a participação comunitária nas políticas de Educação Básica e saúde, nos três níveis de governo. ${ }^{15}$

A adesão dos municípios ao programa acontece a partir da assinatura do Termo de Compromisso entre as Secretarias Municipais de Saúde e de Educação, para então serem determinadas as responsabilidades intersetoriais do PSE, pelos gestores com um conjunto de metas anuais que vinculam as ESF às escolas do território de responsabilidade. Estas metas são de responsabilidade do Grupo de Trabalho Intersetorial Municipal (GTI - M), e monitoradas por intermédio do Sistema Integrado de Monitoramento Execução e Controle (SIMEC). As ações do PSE são desenvolvidas realizadas pelas Equipes da ESF nos territórios, segundo a área de abrangência, o que possibilita o vínculo da educação com os equipamentos públicos da saúde. ${ }^{16}$

Neste programa, as equipes de Saúde da Família constituem, junto com a Educação Básica, uma estratégia para a integração e a articulação permanente entre as políticas e ações de educação e de saúde, com a 
participação da comunidade escolar. Possui ações com base em cinco componentes: a avaliação das condições de saúde das crianças, adolescentes e jovens das escolas públicas; promoção da saúde e ações de prevenção de doenças e de agravos à saúde; educação continuada e capacitação dos profissionais da educação e da saúde e de jovens; monitoramento e avaliação da saúde dos estudantes; monitoramento e avaliação do programa. As ações são constituídas a partir da avaliação clínica, promoção da alimentação saudável, avaliação da saúde e higiene bucal, atualização e controle do calendário vacinal dos alunos entre outras. ${ }^{1,17}$

O Ministério da Saúde compreende que as ações educativas na fase escolar são mais produtivas, uma vez que os escolares são mais receptivos, sendo fundamental se trabalhar saúde na perspectiva da promoção. Nesta fase de vida, o ser humano requer maior atenção pelo processo de crescimento e desenvolvimento físico e psicológico, muitas vezes marcados pela vulnerabilidade. Para isso, se faz necessário desenvolver trabaIhos, envolvendo uma equipe multiprofissional, através da interação e troca de conhecimentos, para juntos realizarem estratégias que proporcionem a identificação de problemas aprendizagem e de saúde, assim como ao processo saúde-doença. ${ }^{9}$

Quanto ao desenvolvimento das ações dos profissionais da saúde e de educação no PSE, espera-se que propiciem uma atitude permanente de empoderamento nos estudantes, professores e funcionários das escolas, princípio básico da promoção da saúde. Para isso, se faz necessário desenvolver, em cada um, a capacidade de atuar de modo a incorporar atitudes e/ou comportamentos adequados para a melhoria da qualidade de vida, a partir da interpretação do cotidiano, tendo como ponto de partida "o que eles sabem" e "o que eles podem fazer", desenvolvendo a autonomia e as competências para o exercício pleno da cidadania. ${ }^{15}$

Entretanto, em alguns municípios, a implementação de políticas sociais com propostas inovadoras e com programas de desenho intersetorial contam com falhas e deficiências e, muitas vezes, não se adequam às prioridades concretas de cada escola. A resistência a mudanças quanto aos modelos de serviço e as burocracias contribuem para o insucesso dessas experiências, devido à predileção pela reprodução de modelos já estabelecidos, sendo a intersetorialidade um objetivo ainda a ser alcançado. Torna-se indispensável à elaboração de planos de estudo que sejam convergentes com as necessidades que apresentam as escolas. Nesse contexto, a Política Nacional de Atenção Básica (PNAB), a Política Nacional de Promoção da Saúde e o PSE, têm contribuído para a criação de um cenário favorável ao debate acerca de uma política nacional de saúde na escola. ${ }^{2,14}$

Dificuldades que permeiam a promoção da saúde no âmbito escolar

A proposta de Escolas Promotoras de Saúde (EPS), desenvolvida na década de 1990 com o conceito baseado na Carta de Ottawa, foi tida como um dos desdobramentos do movimento da promoção da saúde, aspirando uma educação voltada para a saúde integral, através da mudança do paradigma tradicional da saúde escolar. Baseada no estímulo a estilos de vida saudáveis em toda a comunidade escolar, como também o desenvolvimento de um ambiente saudável através de ações de serviços e educação em saúde. ${ }^{2}$

Entretanto, a efetividade da promoção da saúde é um desafio metodológico e político que possibilita gerar conhecimento de programas adaptados à realidade de cada contexto, com vistas à aplicação de metodologia adequada. Segundo alguns autores, espera-se que os atores que constituem a comunidade educativa possam identificar, controlar ou modificar os fatores que condicionam ou determinam a saúde individual e coletiva, assim como a mudança na percepção da situação de saúde, como resultados de efetividade, após a implantação do programa. ${ }^{11,18}$

Nessa perspectiva, a partir das novas compreensões sobre a temática Promoção da saúde foram sendo arquitetados programas de saúde escolar ao longo das décadas. Porém, as ações que têm se destacado são as ações curativas e assistenciais e as ações de promoção têm sido confundidas com as de prevenção, por mais que o conceito de saúde tenha sido ampliado, não conseguindo se desvencilhar das influências do antigo conceito. ${ }^{2}$ Estes problemas específicos são pautados também na necessidade de superação dos modelos tradicionais de ensino, que dão prioridade para a transmissão de conteúdos de forma acrítica, sem evidenciar as contradições ou dúvidas que colaboram para o avanço do conhecimento. ${ }^{19}$

As principais barreiras apontadas ao desenvolvimento das escolas promotoras de saúde foram a infraestrutura inadequada ou insuficiente; a escassez de recurso humano e financeiro; a dificuldade em trabalhar intersetorialmente; a precariedade do apoio político como elemento central nos programas públicos. ${ }^{11}$ Hernandez, ${ }^{20}$ ao realizar estudo sobre o desenvolvimento das Escolas Promotoras de Saúde colombianas identificou que das 32 escolas estudadas, duas não apresentaram nenhum resultado em relação ao aspecto organizacional da estratégia proposta. Apontou como dificuldades a não facilitação por parte de alguns componentes na organização e no funcionamento da equipe gestora, assim como, mudanças de local de trabalho ou retiro laboral do corpo docente e gestor, durante a execução do projeto. Ainda, Gutierrez e Gomez ${ }^{21}$ apontaram como fatores limitantes do programa de intervenção a falta de recursos na execução dos processos e a falta de compromisso da Secretaria de Educação, assim como o desinteresse de alguns pais na participação das atividades propostas, reforçando a importância da união social nos processos de transformação.

No tocante à política de saúde escolar, o PSE apesar de propor uma atuação que rompe com o caráter puramente assistencialista das ações, através de propostas com trabalho direcionado à promoção da saúde, e a intersetorialidade como mecanismo central dessas ações, apresenta também importantes entraves. São apontadas como uma das problemáticas, inconsistências entre a concepção de promoção à saúde adotada na construção do PSE por parte dos profissionais, que desenvolvem ações com uma visão muito limitada, resumindo-a ao desenvolvimento de hábitos alimentares e de higiene. A compreensão quanto ao conceito de pro- 
moção da saúde pelos profissionais é apontada como um grave problema que compromete seu alcance. ${ }^{22-24}$

As fragilidades no PSE, segundo Cavalcanti, Lucena e Lucena, ${ }^{2}$ partem já de inconsistências entre a concepção de promoção à saúde adotada na construção do PSE e a concepção da Organização Mundial da Saúde (OMS). Estes autores referem que, tanto nas análises do Decreto $n^{\circ} 6.286$ de 5 de dezembro de 2007, que cria o PSE, como no Caderno de Atenção Básica Saúde na Escola de 2009, quando estes documentos se reportam à Promoção da Saúde/PS, não assumem claramente a base conceitual da OMS. Os documentos apresentam interpretações reducionistas da abordagem assumida na Carta de Ottawa, em 1986, pela OMS, em que a promoção da saúde é o "processo de capacitação da comunidade para atuar na melhoria da sua qualidade de vida e saúde, incluindo maior participação no controle desse processo".

O Caderno de Atenção Básica Saúde na Escola traz, como desafios mais importantes, as ações de promoção da saúde na escola, a identificação e a vigilância de práticas de risco; a integração com ensino de competência para a vida em todos os níveis escolares; a instrumentalização técnica dos professores e funcionários das escolas e dos profissionais da Estratégia de Saúde da Família, para apoiar e fortalecer as iniciativas; o monitoramento e a avaliação da efetividade das iniciativas, para melhorar o compromisso das escolas com a promoção da saúde de seus alunos, professores e outros membros da comunidade escolar. ${ }^{15}$ Nestes documentos são mencionadas ações profissionais, projetadas através de campanhas sazonais como caminhada e exercícios de alongamento; palestras educativas e oficinas com adolescentes; grupo de ajuda mútua; escovação supervisionada com aplicação tópica de flúor e triagem de risco; palestras educativas; trocas de receitas e encaminhamentos; atividades esportivas; aferição de peso e altura, entre outras. ${ }^{2}$

Estas ações são trazidas como se fora a tradução material do processo mais complexo da Promoção à Saúde sinalizada pela OMS, ratificados por aspectos com responsabilização do setor público, com a participação popular e redução das iniquidades, sem os quais se torna inexequível promover saúde. Os fatores a serem enfrentados pelo PSE não consideram o reconhecimento de que as escolas públicas e seus entornos apresentam demandas para além do que está disposto no componente, sendo tratados apenas como educação em saúde. Essa ambígua compreensão é prejudicial na implementação de programas sociais. ${ }^{2}$ Ferreira et al. ${ }^{16}$ 2012, em seu estudo, revelou que a participação da Educação e da Saúde Escola não é equânime, apontando maior participação da Saúde em áreas de coordenação da Comissão Intersetorial de Educação e Saúde na Escola, assim como no financiamento das ações e centralização no processo de adesão, revelando precariedade das articulações e fragilidade do diálogo intersetorial.

O arcabouço conceitual da promoção da saúde traz novo referencial em que iniciativas mais reflexivas e dialógicas são centrais, a partir da experiência prática dos atores. Observou-se que ainda há a ênfase em fatores comportamentais em detrimento dos fatores de ordem contextual como inferem as proposições da Nova
Promoção da Saúde, ou seja, não se constitui como elemento central do Programa. Propostas impostas de cima para baixo e muito centradas acabam sendo entraves na necessária troca de saberes e experiência entre os dois setores e, ainda, tendem a provocar resistência dos profissionais. ${ }^{1,2}$

\section{CONSIDERACÕES FINAIS}

Observou-se que para as ações no ambiente escolar se concretizarem de forma eficaz e permanente, é necessário que haja comprometimento de todos os envolvidos, propondo o empoderamento dos escolares, professores, funcionários e comunidade escolar. A efetividade da promoção da saúde no ambiente escolar ainda é um desafio, considerando a possibilidade de gerar ações adaptadas às realidades e demandas de cada contexto. Neste aspecto, evidenciam-se como dificuldades limitantes destas ações a resistência ao trabalho intersetorial, baixa adesão à interdisciplinaridade, falta de recursos na execução dos processos e desinteresse dos integrantes da equipe. Ressalta-se, portanto, que as ações de promoção da saúde a serem desenvolvidas devem ser cultivadas de forma contínua e diversificada, propondo interações e mudanças no contexto escolar.

\section{REFERÊNCIAS}

1. Silva CS, Bodstein RCA. Referencial teórico sobre práticas intersetoriais em Promoção da Saúde na Escola. Ciência \& Saúde Coletiva 2016;21(6):1777-88. doi: 10.1590/141381232015216.08522016

2. Cavalcanti PB; Lucena CMF; Lucena PLC. Programa Saúde na Escola: interpelações sobre ações de educação e saúde no Brasil. Textos \& Contextos 2015;14(2):387-402. doi: 10.15448/1677-9509.2015.2.21728.

3. Figueiredo TAM, Machado VLT, Abreu MMS. A Saúde $\mathrm{Na}$ Escola: um breve resgate histórico. Ciênc. Saúde Coletiva 2010;15(2):397-402. doi: 10.1590/S141381232010000200015.

4. Traverso-Yépez MA. Dilemas na promoção da saúde no Brasil: reflexões em torno da política nacional. Interface 2007;11(22):223-38. doi: 10.1590/S141432832007000200004.

5. Czersnia D. O Conceito de saúde e a diferença entre prevenção e promoção. Cadernos de Saúde Pública 1999;15(4),701-10. doi: 10.1590/S0102-311X1999000400004.

6. Carvalho SR. Saúde coletiva e promoção da saúde: sujeito e mudanças. São Paulo: Hucitec, 2005. 174 p.

7. Adelaide- Declaração de Adelaide- A Declaração de Adelaide sobre a Promoção aa Saúde. II Conferencia Internacional De Promoção Da Saúde. Austrália, 1988. Disponível em: < http://www.opas.org.br/declaracao-de-adelaide/>.

8. Gomes LC. O desafio da intersetorialidade: a experiência do Programa Saúde na Escola (PSE) em Manguinhos, no município do Rio de Janeiro. 2012. 173 f. Dissertação (Mestrado em Saúde Pública). Fundação Oswaldo Cruz, Escola Nacional de Saúde Pública Sérgio Arouca, Rio de Janeiro, 2012.

9. Pereira CDFD, Tourinho FSV, Ribeiro JLS, Medeiros SB, Santos VEP. Padrões funcionais de saúde: diagnósticos de enfermagem em escolares da rede pública. Texto Contexto 
Enferm 2013;22(4):1056-63. doi: 10.1590/S0104 07072013000400023

10. Moll J, Rabelo MKO. (Re)conhecendo o território escolar e mapeandoosentidodoser-jovem:contribuiçõesdosprogramas e projetos intersetoriais para superação das vulnerabilidades do escolar. Ciência Saúde Coletiva 2010;15(Supl. 2):3020-6. doi: 10.1590/S1413-81232010000800005.

11. Afonso CMC, Tavares MFL, Luiza VL. Escolas promotoras da saúde na América Latina: uma revisão do período 19962009. Rev Bras Prom Saúde, Fortaleza 2013;26(1):117-127. doi: $10.5020 / 2642$.

12. Mont'Alverne DGB, Catrib AMF. Promoção da saúde e as escolas: como avançar. Rev Bras Promoc Saude 2013,26(3): 307-308. doi:10.5020/2924

13. Guimarães G, Aerts D, Câmara SG. A escola promotora da saúde e o desenvolvimento de habilidades. Diaphora | Revista da Sociedade de Psicologia do Rio Grande do Sul 2012;12(2):88-95

14. Silva CS, Pantoja AF. Contribuições da avaliação na identificação de efetividade da promoção da saúde na escola no município do Rio de Janeiro. B. Téc. Senac: a R. Educ. Prof 2009;35(2):36-49.

15. Brasil. Ministério da Saúde. Secretaria de Atenção à Saúde. Departamento de Atenção Básica. Saúde na escola. Brasília: Ministério da Saúde, Cadernos de Atenção Básica ; n. 24, 2009, p. 96

16. Ferreira IRC, Vosgerau DSAR, Moysés SJ, Moysés ST. Diplomas Normativos do Programa Saúde na Escola: análise de conteúdo associada à ferramenta ATLAS TI. Ciênc. saúde coletiva 2012;17(12):3385-98. doi: 10.1590/S1413 81232012001200023.

17. Cord D, Gesser M, Nunes ASB, Storti MMT. As Significações de Profissionais que Atuam no Programa Saúde na
Escola (PSE) Acerca das Dificuldades de Aprendizagem: Patologização e Medicalização do Fracasso Escolar. Psicologia: Ciência e Profissão 2015;35(1):40-53. doi: 10.1590/1982-3703000952013.

18. Ippolito-Shepherd J. Las escuelas Promotoras de la salud en America Latina: Resultados de la primeira encuesta regional. Washington: OPS; 2005. (Serie Promoción da la salud).

19. Ilha PV, Lima APS, Rossi DS, Soares FAA. Intervenções no ambiente escolar utilizando a promoção da saúde como ferramenta para a melhoria do ensino. Ensaio Pesquisa em Educação em Ciências 2014;16(3):35-54. doi: 10.1590/198321172014160302.

20. Hernandez J. Estrategia escuelas promotoras de la salud em instituiciones de básica primaria de Bucaramanga. Revist Univ Santander Salud 2007;3(39):143-51.

21. Gutierrez AM, Gomez OL. Evaluacion de processo de la estratégia escuelas saludables em la zona urbana del municipio de Cali. Colômbia Médica 2007;4(38):386- 94.

22. Silva KL, Rodrigues AT. Ações intersetoriais para promoção da saúde na Estratégia Saúde da Família: experiências, desafios e possibilidades. Revista Brasileira de Enfermagem 2010;63(5):762-9. doi: 10.1590/S003471672010000500011

23. Faria FHP, Aguiar AC, Moura ATMS, Souza LMBM, Percepções de profissionais de saúde da família e de educação sobre a Promoção da Saúde no ambiente escolar. Revista de APS 2013;16(2):158-64.

24. Closs TT, Collioni AG, Faccioli LS, Lewgoy LB, Leffa LM, Oliveira RS. Articulação intersetorial entre atenção básica e educação: a escola como espaço de promoção de saúde. Anais. Seminário Internacional sobre Políticas Públicas, Intersetorialidade e Família. Desafios éticos no ensino, na pesquisa e na formação profissional; v.1, 2013.

Como citar: COUTO, Analie Nunes et al. O ambiente escolar e as ações de promoção da saúde. Cinergis, Santa Cruz do Sul, v. 17, out. 2016. ISSN 2177-4005. Disponível em: <https://online.unisc.br/seer/index.php/cinergis/article/view/8150>. Acesso em: 11 out. 2016. doi:http://dx.doi.org/10.17058/cinergis.v17i0.8150. 